\title{
Antibiotic use in nasal bone fracture: a single-center retrospective study
}

\author{
Ji Hyuk Jung, \\ Yeo Reum Jeon, \\ Joon Ho Song, \\ Seum Chung \\ Department of Plastic and \\ Reconstructive Surgery, National Health \\ Insurance Service Ilsan Hospital, \\ Goyang, Korea
}

\begin{abstract}
Background: Prophylactic antibiotics are used to prevent surgical wound infection; however, proper indications must be followed with careful consideration of the risks and benefits, especially in clean or clean-contaminated wounds. Nasal bone fractures are the most common type of facial bone fracture. The most common method for treating nasal bone fracture is closed reduction, which is performed inside the nasal cavity without an incision. The purpose of this study was to determine the need for antibiotic use in the closed reduction of nasal bone fractures.

Methods: A retrospective study was conducted using data from the National Insurance Service Ilsan Hospital of the Republic of Korea between 2016 and 2018. The records of patients who underwent closed reduction of nasal bone fracture were reviewed and classified according to sex, age, comorbidities, perioperative antibiotic usage, postoperative complications, nasal packing, anesthesia type, surgeon's specialty, and operation time.

Results: Among the 373 patients studied, the antibiotic prescription rate was $67.3 \%$. Just $0.8 \%$ of patients were prescribed preoperative antibiotics only, $44.0 \%$ were prescribed postoperative antibiotics only, and $22.5 \%$ were prescribed both preoperative and postoperative antibiotics. There were no cases that satisfied the definition of "surgical site infection." Furthermore, 2.1\% of infection-related complications (e.g., mucosal swelling, synechia, and anosmia) occurred only in the antibiotic usage group. The use of nasal packing, anesthesia type, and surgeon's specialty did not show any difference in infection-related complication rates.

Conclusion: According to the study findings, the routine use of perioperative antibiotics is not recommended in uncomplicated nasal bone fracture surgery.
\end{abstract}

Abbreviations: DM, diabetes; ENT, otorhinolaryngology; HTN, hypertension; PCO, polycystic ovaries; Postop, postoperative period; Preop, preoperative period; PS, plastic surgery

Keywords: Antibiotic prophylaxis / Closed fracture reduction / Nasal bone

\section{INTRODUCTION}

Prophylactic antibiotics are used to prevent surgical wound infections. Surgery of facial bone fracture is considered a clean or

\footnotetext{
Correspondence: Seum Chung

Department of Plastic and Reconstructive Surgery, National Health Insurance Service Ilsan Hospital, 100 Ilsan-ro, Ilsandong-gu, Goyang 10444, Korea

E-mail: schung@nhimc.or.kr

This work was supported by National Health Insurance Service Ilsan Hospital (grant No. NHIMC2021-CR-009).
}

Received October 22, 2021 / Revised November 29, 2021 / Accepted December 14, 2021 clean-contaminated wound; therefore, controversy regarding the routine usage of antibiotics is still ongoing. Currently, there are no specific guidelines for antibiotic usage in facial bone fracture surgery, which has led surgeons to prescribe a variety of antibiotics in differing regimens when surgery of facial bone fracture is performed [1-4].

Nasal bone fracture is the most common type of facial bone fracture, accounting for approximately $40 \%$ of facial bone fracture cases. Closed reduction of nasal bone fracture is performed inside the nasal cavity using surgical instruments without an 
incision. Previously reported complications related to nasal bone fracture reduction are limited to dissatisfaction and postoperative nasal deformities [5-9]. Complications related to infection, such as local infection or septicemia, are rarely reported, thus challenging the rationale for the routine use of antibiotics $[6,10]$.

We reported the usage of antibiotics in over half of nasal bone fracture cases treated with closed reduction in a nationwide population-based cohort study in the Republic of Korea [11].

Several factors should be considered when prophylactic antibiotics are administered; bacterial resistance to overuse of antibiotics, increase in health care costs, and risk of antibiotic-related allergic reactions. Thus, antibiotics should only be used when the benefit exceeds other factors $[12,13]$.

This study aimed to evaluate the need for prophylactic antibiotics in the closed reduction of nasal bone fractures. Considering the low infection rate of closed reduction of nasal bone fracture, we hypothesized that perioperative antibiotics would provide no benefit in preventing postoperative wound infection.

\section{METHODS}

A retrospective review was performed using the electronic medical records of patients who underwent closed reduction of nasal bone fractures at the National Insurance Service Ilsan Hospital, Republic of Korea, between January 2016 and December 2018. The study was approved by the Institutional Review Board of the National Insurance Service Ilsan Hospital (IRB No. 2021-03-052). Exclusion criteria were as follows: (1) patients with incomplete medical records; (2) patients who were treated for additional diagnoses other than nasal bone fracture, such as traumatic brain injuries; and (3) patients who had undergone co-operations other than closed reduction of nasal bone fracture.

The following patient information were included in the analysis: sex, age, details regarding perioperative antibiotics, duration of surgery, type of anesthesia, department of surgery in charge, nasal packing, and postoperative complications. The patients were followed up for at least 2 weeks to assess complications, specifically wound infections. According to the United States Centers for Disease Control guidelines, surgical site infections are defined as infections occurring near the incision site [14]. Symptoms including pain or tenderness, localized swelling, erythema, and more serious complications including purulent drainage, presence of abscess, wound dehiscence, or positive microbiologic confirmation were also considered as surgical site infections $[13,15,16]$. There were some cases that partially satisfied the definition of a surgical site infection, indicating functional problems such as mucosal swelling, synechia, and anosmia. We termed these cases with additional antibiotic prescriptions or functional problems as "infection-related," as they can occur as a sequela of postoperative inflammation.

We statistically analyzed demographic differences and baseline differences according to the usage of antibiotics using Student $t$ test. To determine factors independently associated with infection, we performed an analysis of patients with and without any signs of infection using multiple logistic regression, which included the following independent factors: age, sex, operation time, surgeon specialty, type of anesthesia, nasal packing, and comorbidities such as hypertension and diabetes. Statistical significance was set at $p<0.05$. Statistical analyses were performed using SPSS version 23.0 (IBM Corp., Armonk, NY, USA).

\section{RESULTS}

During the study period, 403 patients underwent closed reduction of nasal bone fracture. A total of 373 patients met the inclusion criteria for analysis: mean \pm standard deviation age, $28.6 \pm 16.0$ years; 256 men (68.6\%) and 117 women (31.4\%).

The percentage of antibiotic use during the perioperative period of closed reduction was $67.3 \%$ (patients who received perioperative antibiotics, $n=251$ ), and there were no significant dif-

Table 1. Patient demographics

\begin{tabular}{|c|c|c|c|}
\hline \multirow{2}{*}{ Variable } & \multicolumn{2}{|c|}{ Perioperative antibiotics } & \multirow{2}{*}{$p$-value } \\
\hline & No $(n=122)$ & Yes $(n=251)$ & \\
\hline Sex & & & 0.48 \\
\hline Male & $84(68.9)$ & $172(68.5)$ & \\
\hline Female & $38(31.1)$ & 79 (31.5) & \\
\hline Age (yr) & & & 0.95 \\
\hline Mean \pm SD & $27.7 \pm 15.5$ & $28.9 \pm 16.3$ & \\
\hline Range & $(4-76)$ & $(4-74)$ & \\
\hline Nasal packing & & & $<0.001^{\mathrm{a})}$ \\
\hline Yes & $4(3.3)$ & $121(48.2)$ & \\
\hline Type of anesthesia & & & 0.52 \\
\hline General & $102(83.6)$ & $203(80.9)$ & \\
\hline Local & $20(16.4)$ & 48 (19.1) & \\
\hline Specialty & & & $<0.001^{\mathrm{a})}$ \\
\hline PS & 117 (95.9) & $190(75.7)$ & \\
\hline ENT & $5(4.1)$ & $61(24.3)$ & \\
\hline Hypertension & & & 0.65 \\
\hline Yes & $5(4.1)$ & $13(5.2)$ & \\
\hline Diabetes & & & 0.12 \\
\hline Yes & $1(0.8)$ & $9(3.6)$ & \\
\hline
\end{tabular}

Values are presented as number (\%) unless otherwise indicated. PS, plastic surgery; ENT, otorhinolaryngology.

${ }^{\text {a) }} p$-value $<0.05$. 
Table 2. Patients with infection-related symptoms

\begin{tabular}{|c|c|c|c|c|c|c|c|c|c|}
\hline No. & Age (yr) & Sex & Specialty & Comorbidities & Nasal packing & $\begin{array}{c}\text { Prophylactic } \\
\text { antibiotics }\end{array}$ & Anesthesia & Symptoms & Duration (day) \\
\hline 1 & 8 & $M$ & ENT & Asthma & None & Preop+Postop & General & Prolonged mucosal swelling & 9 \\
\hline 2 & 16 & M & ENT & None & None & Postop & Local & Nasal synechia & 32 \\
\hline 3 & 20 & $\mathrm{~F}$ & PS & None & None & Postop & General & Nasal synechia & 30 \\
\hline 4 & 29 & $\mathrm{~F}$ & ENT & PCO, alcoholic liver disease & Visco & Preop+Postop & General & Prolonged mucosal swelling & 22 \\
\hline 5 & 37 & $\mathrm{~F}$ & PS & None & None & Postop & General & Prolonged mucosal swelling & 15 \\
\hline 6 & 46 & $\mathrm{~F}$ & PS & None & Merocel & Postop & General & Nasal synechia & 60 \\
\hline 7 & 56 & M & PS & HTN, DM, liver cirrhosis & Merocel & Postop & General & Prolonged mucosal swelling & 11 \\
\hline 8 & 63 & $\mathrm{~F}$ & PS & HTN, dyslipidemia & None & Preop+Postop & General & Prolonged mucosal swelling, anosmia & 30 \\
\hline
\end{tabular}

M, male; F, female; ENT, otorhinolaryngology; PS, plastic surgery; PCO, polycystic ovaries; HTN, hypertension; DM, diabetes; Preop, preoperative period; Postop, postoperative period.

ferences in sex and age between antibiotic usage and non-usage groups. Second-generation cephalosporins was prescribed in all the antibiotic usage cases. Nasal packing was performed in 125 patients (33.5\% of total patients) and the longest duration of nasal packing was 4 days. Antibiotics were prescribed more often when nasal packing was performed $(p<0.01)$. There was no significant difference in antibiotic administration rates according to the type of anesthesia or comorbidities (Table 1). Eight patients in the antibiotic usage group presented with infectionrelated symptoms, but no patients in the non-usage group had any infection-related symptoms. In the antibiotic usage group, three patients were prescribed additional oral antibiotics due to prolonged mucosal swelling over a week after surgery. In addition, three patients were diagnosed with nasal synechia within a month of the operation, and one patient was diagnosed with anosmia. There was no significant difference in the incidence of "infection-related symptoms" between surgeons (Table 2).

The multiple regression analysis showed no significant differences in rates of infection-related symptoms for usage of perioperative antibiotics, age, sex, nasal packing, type of anesthesia, surgical specialty or operation time. Only patients with hypertension showed a higher risk of symptoms (odds ratio, 5.67) (Table 3).

\section{DISCUSSION}

Closed reduction of nasal fracture is performed without an incision and it is less traumatic than other operations performed inside the nasal cavity such as septoplasty. Closed reduction is also considered a simpler operation, with a shorter operation time than septoplasty. However, we reported the usage of antibiotics in over half of nasal bone fracture cases treated with closed reduction in a nationwide population-based cohort study in the Republic of Korea, which raised the need for research concerning necessity of antibiotics [11].
Table 3. Factors associated with infection-related symptoms

\begin{tabular}{|c|c|c|c|c|}
\hline \multirow{2}{*}{ Factor } & \multicolumn{2}{|c|}{ Infection-related symptoms } & \multirow{2}{*}{$p$-value } & \multirow{2}{*}{ Odds ratic } \\
\hline & No $(n=365)$ & Yes $(n=8)$ & & \\
\hline Prophylactic antibiotics & & & 0.22 & 0.50 \\
\hline Yes & $244(66.8)$ & $8(100)$ & & \\
\hline No & $121(33.2)$ & 0 & & \\
\hline Sex & & & 0.06 & 3.89 \\
\hline Male & 253 (69.3) & $3(37.5)$ & & \\
\hline Female & $112(30.7)$ & $5(62.5)$ & & \\
\hline Age (yr) & $28.4 \pm 15.6$ & $37.9 \pm 16.5$ & 0.10 & 1.01 \\
\hline Nasal packing & & & 0.08 & 2.44 \\
\hline No & $245(67.1)$ & $3(37.5)$ & & \\
\hline Yes & $120(32.9)$ & $5(62.5)$ & & \\
\hline Type of aesthesia & & & 0.67 & 7.10 \\
\hline General & 298 (81.6) & $7(87.5)$ & & \\
\hline Local & $67(18.4)$ & $1(12.5)$ & & \\
\hline Specialty & & & 0.58 & 0.43 \\
\hline PS & 301 (82.5) & $6(75.0)$ & & \\
\hline ENT & $64(17.5)$ & $2(25.0)$ & & \\
\hline Hypertension & & & $0.01^{\mathrm{a})}$ & 5.67 \\
\hline Yes & $16(4.4)$ & $2(25.0)$ & & \\
\hline Diabetes & & & 0.68 & 2.40 \\
\hline Yes & $10(2.7)$ & 0 & & \\
\hline Operation time (min) & $10.0 \pm 4.6$ & $10.5 \pm 5.7$ & 0.74 & 1.06 \\
\hline
\end{tabular}

Values are presented number (\%) or mean $\pm \mathrm{SD}$.

PS, plastic surgery; ENT, otorhinolaryngology.

${ }^{\text {a) }} p$-value $<0.05$.

We attempted to use the definition of "surgical site infection" from the National Healthcare Safety Network, Centers for Disease Control and Prevention [10]; however, there were no cases that fully satisfied these criteria. Therefore, we included several symptoms and functional problems that may represent the sequelae of an infection and termed them "infection-related" symptoms. Nonetheless, only $2.14 \%$ of cases ( 8 out of 373 patients) were classified as infection-related symptoms, and the 
incidence of infection-related symptoms was not related to the use of antibiotics. The findings of this study demonstrate that the incidence of infection-related complications in closed reduction of nasal bone fracture is very low, therefore the routine usage of perioperative antibiotics is not indicated.

The strength of this report is that this may be the first to study the necessity of perioperative antibiotics in closed reduction of nasal bone fracture. However, there are also several limitations. First, although we analyzed six surgeons' data from two departments, there was little diversity of cases because the retrospective study was done at a single hospital. Second, it is difficult to determine if mucosal swelling and synechia are direct sequalae of postoperative inflammation. Nevertheless, considering that these symptoms only occurred in the antibiotic usage group and not in the no-usage group, it is supportive of the statement that there is a lack of evidence for the routine use of perioperative antibiotics in closed reduction of nasal bone fracture. Also, the result showed a higher infection-related symptom rate when patients were diagnosed with hypertension; however, as there were only a few such cases, further large-population study is needed on high-risk comorbidities related to surgical site infection. Finally, although our analysis considered patient comorbidity (e.g., diabetes and hypertension), we did not consider factors that could affect the patient's nasal mucosal condition, such as smoking history or malnutrition. In addition, if there is bleeding during the operation, there is a possibility of mucosal injury. Although there is debate about usage of antibiotics when there is mucosal injury or epistaxis, because we did not compare the incidence of infection according to degree of bleeding during surgery, surgeons may consider the usage of prophylactic antibiotics according to the patient's mucosal condition as well as possibility of mucosal injury during operation [17-20].

Ideal surgical prophylactic antibiotics should prevent operation-related infections and complications with less adverse effects such as outbreaks of antibiotic-resistant species. Considering this point, along with the cost of healthcare, the shortest usage of effective antibiotics is optimal [18-21]. It is the responsibility of physicians to use antibiotics judiciously and effectively based on the most current literature. Restrictive antibiotic usage is desirable for short-term use in specific situations with clear evidence.

This study does not support the routine use of perioperative antibiotics in uncomplicated nasal bone fracture surgery. The prevalence of postoperative wound infection reported in this retrospective study after closed reduction of nasal bone fracture was minimal, and there was no significant benefit in prescribing antibiotics perioperatively to prevent infections after reduction of nasal bone fracture. A specific guideline and establishing consensus by further large-sized study with a multicenter analysis are required.

\section{NOTES}

\section{Conflict of interest}

No potential conflict of interest relevant to this article was reported.

\section{Ethical approval}

The study was approved by the Institutional Review Board of National Insurance Service Ilsan Hospital (IRB No. 2021-03052) and performed in accordance with the principles of the Declaration of Helsinki. The informed consent was waived because this study design is the retrospective electronic medical records.

\section{ORCID}

$\begin{array}{ll}\text { Ji Hyuk Jung } & \text { https://orcid.org/0000-0002-8556-3413 } \\ \text { Yeo Reum Jeon } & \text { https://orcid.org/0000-0002-3435-753X } \\ \text { Joon Ho Song } & \text { https://orcid.org/0000-0002-0328-848X } \\ \text { Seum Chung } & \text { https://orcid.org/0000-0003-3273-6787 }\end{array}$

\section{Author contribution}

Conceptualization: Yeo Reum Jeon, Seum Chung. Data curation: Ji Hyuk Jung, Yeo Reum Jeon, Seum Chung. Formal analysis: Yeo Reum Jeon, Seum Chung. Methodology: Yeo Reum Jeon. Project administration: Seum Chung. Visualization: Ji Hyuk Jung, Yeo Reum Jeon. Writing - original draft: Ji Hyuk Jung. Writing - review \& editing: Ji Hyuk Jung, Yeo Reum Jeon, Seum Chung. Investigation: Yeo Reum Jeon. Supervision: Yeo Reum Jeon, Joon Ho Song, Seum Chung.

\section{REFERENCES}

1. Mundinger GS, Borsuk DE, Okhah Z, Christy MR, Bojovic B, Dorafshar AH, et al. Antibiotics and facial fractures: evidencebased recommendations compared with experience-based practice. Craniomaxillofac Trauma Reconstr 2015;8:64-78.

2. Soong PL, Schaller B, Zix J, lizuka T, Mottini M, Lieger O. The role of postoperative prophylactic antibiotics in the treatment of facial fractures: a randomised, double-blind, placebo-controlled pilot clinical study. Part 3: Le Fort and zygomatic fractures in 94 patients. Br J Oral Maxillofac Surg 2014;52:329-33.

3. Zosa BM, Elliott CW, Kurlander DE, Johnson F, Ho VP, Claridge JA. Facing the facts on prophylactic antibiotics for facial fractures: 1 day or less. J Trauma Acute Care Surg 2018;85:44450 . 
4. Brooke SM, Goyal N, Michelotti BF, Guedez HM, Fedok FG, Mackay DR, et al. A multidisciplinary evaluation of prescribing practices for prophylactic antibiotics in operative and nonoperative facial fractures. J Craniofac Surg 2015;26:2299-303.

5. Hwang K, Yeom SH, Hwang SH. Complications of nasal bone fractures. J Craniofac Surg 2017;28:803-5.

6. Han DG. Considerations for nasal bone fractures: preoperative, perioperative, and postoperative. Arch Craniofac Surg 2020;21:3-6.

7. Choi MH, Cheon JS, Son KM, Choi WY. Long-term postoperative satisfaction and complications in nasal bone fracture patients according to fracture type, site, and severity. Arch Craniofac Surg 2020;21:7-14.

8. Park HK, Lee JY, Song JM, Kim TS, Shin SH. The retrospective study of closed reduction of nasal bone fracture. Maxillofac Plast Reconstr Surg 2014;36:266-72.

9. Kim KS, Lee HG, Shin JH, Hwang JH, Lee SY. Trend analysis of nasal bone fracture. Arch Craniofac Surg 2018;19:270-4.

10. Jang N, Shin HW. Are postoperative prophylactic antibiotics in closed reduction of nasal bone fracture valuable?: prospective study of 30 cases. Arch Craniofac Surg 2019;20:89-93.

11. Jeon YR, Jung JH, Song JH, Chung S. Antibiotic use in nasal bone fracture: a nationwide population-based cohort study in Korea. Arch Craniofac Surg 2021;22:254-9.

12. Barie PS. Rational and appropriate use of prophylactic antibiotics. Arch Surg 1997;132:320-1.

13. World Health Organization. Global guidelines on the prevention of surgical site infection. Geneva: World Health Organiza- tion; 2016.

14. Borchardt RA, Tzizik D. Update on surgical site infections: the new CDC guidelines. JAAPA 2018;31:52-4.

15. Horan TC, Andrus M, Dudeck MA. CDC/NHSN surveillance definition of health care-associated infection and criteria for specific types of infections in the acute care setting. Am J Infect Control 2008;36:309-32.

16. Horan TC, Gaynes RP, Martone WJ, Jarvis WR, Emori TG. CDC definitions of nosocomial surgical site infections, 1992: a modification of CDC definitions of surgical wound infections. Infect Control Hosp Epidemiol 1992;13:606-8.

17. Tran QK, Rehan MA, Haase DJ, Matta A, Pourmand A. Prophylactic antibiotics for anterior nasal packing in emergency department: a systematic review and meta-analysis of clinically-significant infections. Am J Emerg Med 2020;38:983-9.

18. Makitie A, Aaltonen LM, Hytonen M, Malmberg H. Postoperative infection following nasal septoplasty. Acta Otolaryngol Suppl 2000;543:165-6.

19. Caniello M, Passerotti GH, Goto EY, Voegels RL, Butugan O. Antibiotics in septoplasty: is it necessary? Braz J Otorhinolaryngol 2005;71:734-8.

20. Georgiou I, Farber N, Mendes D, Winkler E. The role of antibiotics in rhinoplasty and septoplasty: a literature review. Rhinology 2008;46:267-70.

21. Bratzler DW, Dellinger EP, Olsen KM, Perl TM, Auwaerter PG, Bolon MK, et al. Clinical practice guidelines for antimicrobial prophylaxis in surgery. Surg Infect (Larchmt) 2013;14:73-156. 